\title{
Application of the Quantum Theory of Few-Body Scattering for the Calculations of Resonances
}

\author{
S.Pozdneev \\ P.N.Lebedev Physical Institute, Russian Academy of Sciences \\ Moscow, Russia 107924 \\ Email: pozdneev@sci.lebedev.ru
}

\begin{abstract}
The main features of the resonance scattering of electrons by molecules are described. The parameters of the resonances are calculated in the frame of two different approaches: 1) the theory of collisions in a two-body system (where applicable); 2) the quantum theory of scattering in a few-body system based on Faddeev-Yakubovsky equations. The results of calculations of the resonant cross sections of electron and atom collisions with molecules are presented. Obtained results are compared with the available experimental data and with the results of calculations based on other approximations. In addition some biological applications (e.g. properties biopolymer molecules) are presented. Application of the results for determination of biopolymer molecule parameters is presented.
\end{abstract}

Keywords: Calculations of resonances in scattering, Faddeev equations, Efimov states.

\section{Introduction}

Resonances appear in any branch of physics: from the molecular physics to the physics of elementary particles. They offer a variety and peculiarities of physical patterns of phenomena. The concept of resonance is one of the fundamental concepts in quantum physics. One can attach a broad physical meaning to the term resonance, including stable levels and implying their effect on scattering processes $[1,2]$.

Resonances play an important role in all fields of physics. In case of irreversible processes the resonances are responsible for non-integrability of most dynamic systems in accordance with the Poincare theorem [3]. A theoretical explanation of resonances and their parameters can be found by means of the analysis of interaction forces between particles considered as elementary in such processes. For example, resonant processes in atomic physics are determined by the forces of interaction between electrons and nuclei, while resonances in nuclear physics are determined by forces acting between nucleons.

The importance of resonant processes is determined by the fact that all practical applications of experimental studies are based on resonances since they are characterized by large cross sections or long lifetimes as compared to nonresonant processes. Also the resonant processes play an important role in low-temperature plasma (they determine the production and disappearance of excited and charged particles, i.e. determine optical and electrical properties of a plasma), in controlled thermonuclear synthesis, mu-catalysis, etc. [3-14].

For example, in laser physics, molecular reactions, which produce the excited molecules, can be effectively used in chemical lasers if they have a number of the following salient features [14-15]:

1. the thermal effect and rate constants in these reactions should be high enough and

2. a considerable part of the released energy must be transferred to the energy of reaction products.

Thus it is not surprising that the practical demands for extending the spectral range and increasing the power of chemical lasers have boosted an active search for appropriate chemical reactions which can be described accurately and completely on the base of experimental investigations. Therefore, at present the theoretical methods of investigation of elementary processes are attracting considerable attention $[3,5-11,13-15]$

In recent years, the investigations of Efimov physics [1], the universal physics of few particles interacting via nearly resonant short-range interactions, have been developed tremendously, both on the experimental $[1-11]$ and theoretical fronts. The essence of this physics is the appearance of a universal $1 / R^{2}$ attraction 
between three particles at an average separation $R$. This long-range three-body attraction discovered by V. Efimov arises from the pairwise interactions despite their finite range. It can be interpreted as an interaction between two particles mediated by a third particle. Its strength is universally determined by the masses and quantum statistics of the particles. The Efimov attraction extends from distances of order of the interaction range $b$ to distances of order of $|a|$, where $a$ is the scattering length of the pairwise interaction. It therefore requires condition $|a|>b$ that is well satisfied for resonant pairwise interactions. At the unitary limit $a \rightarrow \infty$, the Efimov attraction extends to infinity. Dependence of interaction potential as $1 / R^{2}$ leads to the existence of infinite number of bound states known as the Efimov trimers. Furthermore, each bound state is related to the neighbouring state by a scale transformation due to the scale invariance of $1 / R^{2}$ potentials $[1,2]$, so that the energy spectrum forms a geometric series. This constitutes the most remarkable and characteristic feature of the Efimov trimers. However, since the three particles are attracted to each other, the physics at short separations (comparable to the range $b$ ) fixes the wave functions and spectrum of the Efimov trimers [1-15].

In this paper the results of calculations of resonance processes are presented. The calculations are based on few-body Faddeev equations [13] and are performed in the frame of approximation explained in detail in [14].

\section{Results of Calculations}

We will analyze these peculiar resonant states quantitatively by using the Faddeev integral equations [13] presented in previous papers [13-15].

Let us consider (as an example) the calculation of cross sections for the simplest reaction of dissociative attachment of electrons to hydrogen molecules. The results of this calculation are shown in Fig. 1 together with the latest experimental data $[4,19-21]$ and the results of calculations based on other approximations [5-11]. These results confirm that the resonant states considered above exist in this system. It should be noted that experimental results of observation of three-particle resonant states were presented for the first time in [3] to describe the dissociative attachment of an electron to hydrogen molecules. However, in view of the energy distribution for the electron beam of width $0.1 \mathrm{eV}$ only non-monotonicity of the energy dependence of the dissociative attachment cross section was noted in these experiments; this non-monotonicity was confirmed in theoretical calculations published more than 20 years ago [14, 17]. The oscillatory structure of the dissociative attachment cross sections was confirmed only recently in the experiments [19], in which special technique was used for energy stabilization of the electron beam $(\mathrm{meV})$. The detailed structure of cross section in $\sim 3.75 \mathrm{eV}$ region is presented in Fig. 2.

To estimate the influence of particle charges on the effect, we consider the scattering of electrons by hydrogen halide molecules. Since the electron affinity to the hydrogen atom is much smaller than its affinity to a halogen atom [18], a hydrogen halide molecule can be visualized as a system consisting of a proton and a negative halogen ion. Thus, in the approach proposed here, the main approximation is that the interaction of the projectile electron with the nuclei of the target molecule is replaced by the interaction of the impinging electron with the proton and the negative halogen ion. The complex many-particle problem of calculation of electron scattering cross section by diatomic molecules is reduced to the problem of collision in a three-body system that can be solved by using the method of quantum scattering problem in a few-particle system. Naturally, this approximation is valid for energies of the impinging electron lower than the electron excitation energy of the molecule.

Computational difficulties encountered in calculation of cross sections in the given approximation are mainly associated with the long-range Coulomb interaction potentials between projectile electron, proton, and negative halogen ion. It was mentioned above that in this case the integral Faddeev equations cannot be applied directly; either these equations should be modified, or the differential formulation of the Faddeev equations in the coordinate state should be used [13-15]. It should be noted that in the general case of scattering of an electron from halogen molecules in the given approximation, 


$$
e+\left(H^{+} G^{-}\right) \rightarrow \begin{cases}e+\left(H^{+} G^{-}\right) & \text {elastic scattering processes } \\ e+\left(H^{+} G^{-}\right)^{*} & \text { excitation processes } \\ H^{+}+\left(e, G^{-}\right)^{*} & \text { rearrangment processes } \\ G^{-}+\left(e, H^{+}\right)^{*} & \text { with excitation } \\ e+H^{+}+G^{-} & \text {dissociation processes } \\ e+H+G & \text { dissociation processes } \\ e+e+H^{+}+G & \text { ionization processes }\end{cases}
$$

the Faddeev equations for four mutually interacting bodies (two electrons, a halogen atom, and a proton) should be used. However, for some processes such as dissociative attachment reactions, we can confine our analysis to equations for three pairwise interacting bodies.

In our case, to calculate dissociative attachment of an electron to hydrogen halide molecules, we apply the Faddeev equation for three charged particles in the coordinate space [13-15]

Figures 3 - 6 show the results of calculation of the cross sections of electron dissociative attachment to hydrogen halide molecules and their isotope-substituted modifications in the ground state and in excited vibrational-rotational states as well as experimental results $[4,7,9,11,19,21]$ and the results of calculations based on other approximations [4-11] that demonstrate suppression of oscillations in the scattering cross sections.

Let us consider the effect of these peculiar resonances on the rates of chemical reactions, which is interesting for explaining electron transport in proteins (physically, this transport is one of the main functionally important processes in a cell $[15,23])$. Knowing this transport mechanism, it would be possible to explain the process of transition from structurally disorganized chemical transformations (e.g., in solutions) to coordinated subsequent stages typical for biological systems [15]. It should be noted that no new interactions are used in nature and the process is organized due to an appropriate choice of molecular structures and the corresponding well-known interactions. Thus, identification and analysis of these interactions will make it possible to understand physical mechanisms of processes occurring in proteins and other molecular structures such as DNA and RNA.

The application of the method presented above for the calculation of real biopolymer molecules now encounters many difficulties, namely, absence of reliable spectroscopic data on clusters (in the difference from diatomic and polyatomic molecules $[4,7,23]$ and some others). However, in some particular cases certain estimates can be made. One can prove [23] that under certain condition biopolymers consist of similar elements with spiral structure. This is experimentally confirmed by sublimation of frozen water solutions of organic compounds, including amino acids (protein monomers), nucleotides (DNA and RNA monomers) and their mixtures. In the experiments [23], for solution concentrations exceeding $10^{-2}$ gmol/l, the porous remnants (matrices of crystals of dissolved substances) are formed, while for lower concentrations the structure of matrices changes drastically and they have the form of undirectional tubular lines. The formation of these lines is due to solution freezing which is accompanied by replacement of impurity components in front of the newly formed ice and the component density fluctuation lead to shifts in the ice crystal lattice, i.e. dislocations that form steps. In the course of crystallization the step winds, which is shown schematically in Fig.7. Experiments demonstrated that monomers may joint in lines in the course of their assembly. This is because freezing of water solutions of amino acids of certain concentration initiates an interaction between components (under UV radiation $\lambda=250-300 \mathrm{~nm}$ ) and leads to reaction of biopolymer synthesis. The calculation based on the proposed model shows that the mutual orientation of monomers in chains under self-assembly is due to the specific multi-particle interaction precisely described above, where molecules attach to each other tending to occupy a position with minimal potential energy.

As a result of assembly of $L$-amino acid chains the role of matrix is played by nucleotides and vice versa. This is how one-to-one correspondence between molecules, i.e. a genetic code appears owing to multi-particle dynamic interactions. The estimates obtained on the basis of proposed model show that

- amino acids and nucleotides form their individual chains and practically no restrictions exist for assembly of large $\left(10^{6}-10^{9}\right.$ atoms $)$ molecules of nucleic acid, 
- alternation of regions with periodic and aperiodic character of succession of different monomers in the chains depends on the velocities of molecular motion at different dislocation steps upon freezing,

- not only nucleotides and amino acids but also their isomers, if they were presented in the initial solution, are distributed in independent chains. This proves that L-amino acids that rotate the polarization plane of light to the left form the chain in space with a shape of the right helix and are located near the right helix of nucleotides, while D-amino acids that form the left helix are located across the nucleotide spiral. This fact proves the natural separation of stereo-isomers in the course of evolution [23]. These results, i.e. the formation of ordered helical structures of impurity molecules, or one-dimensional assembly, are directly related to the problem of appearance of life. The practical importance of this phenomenon refers to the creation of biopolymers with prescribed properties and to the possibility of construction of various technical elements of bioelectronics on their basis.

To study the processes described above, we first consider a simple system of two identical coupled particles tunneling through a potential barrier. For this consideration we will use mathematically correct quantum theory of scattering in a few-body system [13-15].

It should be noted that tunneling of particles (including structured ones) is usually considered on the basis of well-known theories [24] and results are automatically extended to many-particle systems (especially in applied studies, e.g., in biology [23]). Most results in these applications are associated precisely with analysis of tunneling through various potential barriers of multicomponent structured complexes. Quite often, such results do not correspond to the initial problem and do not reproduce experimental data. It should be noted that if the barrier size is much larger than the characteristic size of a complex, the difference from the structureless case is insignificant. If the size of the complex is comparable with the barrier width, mechanisms $[15,25]$ leading to anomalous transparency of the barrier appear (analogously to the Ramsauer effect $[4,5,7]$ ) .

The physical reason for the barrier transparency is associated with the possibility of formation of a barrier resonance since the potential energy of the system may have a local minimum providing the metastable state of the complex; to this end, the interaction of all particles of the complex with the barrier is required.

To demonstrate this effect we use the quantum scattering theory. Let us consider the tunneling of a pair of identical particles $m_{1}=m_{2}=m$ coupled by various types of interaction (harmonic oscillator, Morse and Gauss potentials) through a potential barrier which was simulated by a potential of the form $[25]$

$$
\left.V\left(x_{-}^{+} y\right)=a \exp \left(\left(x_{-}^{+} y\right)^{2} / 2 b\right) / \sqrt{(} 2 \pi b\right),
$$

where $a$ and $b$ are the barrier height and width. The results of calculations are shown in Fig.8 . It can be seen from the figure that, for a barrier with height much larger than the characteristic size of the incident complex, its transmission probability differs insignificantly from the transmission probability in the case of structureless particles. If the size of the complex is comparable with the spatial size of the barrier, mechanisms leading to a substantial transparency of the barrier (and even to its total transparency in some cases) appear.

In the simplest case of a complex consisting of two particles, the physical pattern of the barrier transparency is determined by the formation of a resonant state upon the passage of only one of the particles through the barrier. Thus, two particles are on different sides of the barrier (i.e. this resonant state is preserved until the other particle passes through the barrier). The barrier width is determined by the lifetime of this resonance.

It should be noted that the breaking of symmetry of penetrability for structured particles may serve as a possible mechanism explaining different values of penetrability of biomembranes in opposite directions (osmosis). Another explanation of penetrability of a biomembrane is given in [23] on the basis of the assumption that an isothermal phase transition of the melting/crystallization type occurs in a monolayer of the membrane, but in $2 \mathrm{D}$ and not in $3 \mathrm{D}$ system. This makes it possible to interpret some peculiarities of blood circulatory system, which could not been explained earlier.

Analogous results are considered in [24]; however, the results concerning the penetrability of potential barriers for structured particles are completely different.

Figure 8 shows that the tunneling probability in some cases may attain unity, which can be explained by interference suppression of the reflected wave (this phenomenon is widely used for blooming of optical 
systems). As the number of interacting particles increases, the effect of enhancement of the barrier penetrability may substantially increase. Thus, under certain conditions, coupled clusters may not only surmount obstacles more easily, but can also be themselves transparent to other particles (this is often encountered in biological systems).

The most astonishing fact is that this mechanism of potential barrier transparency for structural particles was confirmed in experiments [19-25].

These features are commonly observed in real systems, e.g., in the simplest chemical reactions induced by electrons:

$$
e+A B\left(v_{1}, J_{1}\right) \rightarrow \begin{cases}e+A B\left(v_{1}, J_{1}\right) & \text { - elastic scattering processes } \\ e+A B\left(v_{2}, J_{2}\right) & \text { - vibrational-rotational excitation } \\ A^{-}(e A)+B & \text { - dissociative attachment } \mathrm{A}+\mathrm{eB} \\ A+B^{-}(e B) & \text { of electron to molecule } \\ e+A+B & \text { - dissociation of molecule }\end{cases}
$$

In the approximation of the quantum theory of scattering in a few-body system, it is possible to reproduce the experimental data [9-11, 19-26] for the simplest chemical reactions occurring during the interaction of electrons with diatomic molecules in the ground state as well as in excited vibrationalrotational states. These results are presented in Figs. 9-12; all calculations in this case are performed in the above-mentioned approximation in which the interaction of an electron with nuclei and electrons of the target molecule was replaced by the interaction of the electron with each atom as a whole (the atom was treated as a force center). The same figures also show the results of calculations performed in the resonance model approximation with nonlocal potentials based on the quantum theory of scattering in a two-body system [26].

It should be noted in this connection that the application of nonlocal separable potentials opens wide prospects for detailed reproduction of experimental results $[14,15,17]$. For example, using the separable potential

$$
V\left(k, k^{\prime}\right)=\lambda g(k) g\left(k^{\prime}\right)
$$

with form factors of the type

$$
g_{N L}(k)=k^{L} \sum_{i=0}^{N} \gamma_{i} k^{2 i}\left(\prod_{i=1}^{L+N}\left(1+\beta_{i} k^{2}\right)\right)^{-1} .
$$

we can reproduce the experimental data on scattering cross sections with a preset accuracy both in the approximation of the two-body problem $[10,16]$ and in the approximation of the few-body problem $[14,15,17,27]$.

However, the main difficulty encountered in the application of these potentials is associated with the choice of parameters $\lambda, \gamma$, and $\beta$. For this reason, we use here the simplest pair potentials [15] whose parameters can be chosen on the basis of real spectroscopic data [18] rather than empirical data as was done in [26].

It should be noted once again that this approximation appears as reasonable for energies of the projectile electron lower than the electron excitation energy of the molecule. Otherwise, it is necessary to use the many-particle approximation instead of the three-particle approximation since the contributions from the dissociation channels become significant (this is demonstrated in Figs. 9, 11, and 12). Consequently, we can speak about the agreement with the experimental results only on the average in view of the initial model of the process as well as the simplest pair potentials simulating the interaction of an electron with atoms $[14,15,17]$.

Another thinking demonstrations Faddeev's approach are the calculations of the reactions for threebody recombination $\mathrm{He}+\mathrm{He}+\mathrm{He} \rightarrow \mathrm{He}_{2}+\mathrm{He} \mathrm{Fig} .13$ and $\mathrm{CsBr}+\mathrm{Xe} \rightarrow \mathrm{Cs}^{+}+\mathrm{Br}^{-}+\mathrm{Xe}$ Fig.14, $\mathrm{CsBr}+\mathrm{Hg} \rightarrow \mathrm{Cs}^{+}+\mathrm{Br}^{-}+\mathrm{Hg}$ Fig.15 with pair-wise potentials presented in [29-34].

Comparison of the results of our calculations with the available experimental data [4-11, 19-23] shows that simulation of the electron interaction with each atom of the molecule, based on Eqs. $(1,6)$ that correspond to the multiple scattering pattern $[6,14,17]$, makes it possible to attain satisfactory agreement 
with experiment (coincidence of the orders of magnitude of cross sections, including isotopic effects and threshold singularities [14-17]).

Well-known theoretical methods for studying resonant processes occurring during electron collisions with molecules [4-11] (the boomerang method, the R matrix method, the method of time evolution of the wave function, the Feschbach operator method, etc.) are based on interpreting this process as a multistage process. The first stage involves the electron capture by a molecule and formation of a negative molecular ion. The second stage is the decay (evolution) of this state to various states of the decay products: a negative ion and a neutral or excited atom, two neutral or excited atoms and an electron (dissociation of the molecule), and an excited molecule and an electron (excitation of the molecule by electron impact). The basis of this formalism (i.e. formation of an intermediate state of a negative molecular ion) appears as not always substantiated from the physics point of view. For example, in the case of dissociative attachment of an electron to a hydrogen molecule, the lifetime of this complex is comparable with the electron mean free time, during which it covers a distance equal to the diameter of the hydrogen molecule. An analogous situation also emerges for the reaction [14, 27]

$$
O\left({ }^{3} P\right)+C S\left(X^{1} \Sigma^{+}\right) \rightarrow C O\left(X^{1} \Sigma^{+}\right)+S\left({ }^{3} P\right),
$$

in which a considerable fraction of the translational energy (in accordance with the momentum limit $E_{v} / E_{t} \sim 0.88$ [27]) is transformed into the vibrational energy of the CO molecule. Such a reaction also occurs without the formation of an intermediate complex.

Naturally, there are many reactions in which a long-lived intermediate complex is formed in the course of the reaction (see [5-11, 14, 15, 17] for details). However, for some processes like those described above, a preliminary analysis of experimental data for given processes is required for analyzing various collisions; the absence of such an analysis often leads to erroneous interpretation of experimental data.

Thus, a class of processes existing in atomic, chemical, and biological physics can be referred to as direct processes in analogy with nuclear physics. The main feature of these processes is that no intermediate long-lived complex is formed in the course of scattering.

Consequently, the most adequate methods for interpreting such direct processes and reaction occurring with the formation of an intermediate complex were proposed by Faddeev, Yakubovskii, and Merkur'ev [13], who developed a quantum theory of scattering in few-body systems without model assumptions concerning the formation of an intermediate complex during a collision. This method can be applied for describing direct processes as well as processes occurring with the formation of intermediate long-lived states. Thus, we can state that quantum transparency effects for various barriers and peculiarities of chemical reaction mechanisms described above can take place in various branches of physics, chemistry, and biology and can be interpreted in the frame of non-relativistic quantum mechanics with the help of the formalism proposed in [13-15].

It is especially important for molecular biology, in which a consistent and mathematically correct explanation of fermentation reactions has not been obtained as yet [23]. The contemporary description of these reactions based on the assumption that a part of the free energy released as a result of a reaction is used for accelerating catalysis, i.e. penetration through a barrier (recuperation of energy), does not permit to analyze the reaction energy quantitatively. For this reason, it is extremely difficult to confirm or reject the proposed model experimentally.

On the contrary, the above substantiation of the transparency of potential barriers for structural complexes with a size commensurate with the barrier width provides an explanation for such reactions based on the well-known physical principles in the framework of ordinary quantum theory for a few-body system.

Acknowledgments. The author is indebted to many people of Laboratory of Photochemistry Processes, Theoretical Department P.N.Lebedev Physical Institute, Laboratory Collision Theory Lenigrad State University who enabled his findings partialy described in this paper.

I am most grateful to Professors G.P.Drukarev, Yu.N.Demkov, S.P.Merkuriev, V.Efimov, G.J.Schulz, I.Fabricant, E.A.Soloviev, I.Yurova and many others for initiating this study.

This study was supported by the Taiwan Academy of Sciences (grant no NSC 85-212-M-007-009), Scientific Research Foundation of the China People's Republic (grant no. NSF 19734030), Joint Research 
Foundation of Israel and the USA, and the Russian Foundation for Basic Research (projects nos. 98-0217266 and 01-02-16075).

\section{References}

1. V. Efimov, Influence of Resonance in Pair Forces on the Spectrum of Three-Particle Levels (Mosk. Inzh.-Fiz. Inst., Moscow, 1973) [in Russian].

2. T. Andras, Resonances in Few-Body Systems (Springer,New York, 2002).

3. A. Poincare, Selected Works (Nauka, Moscow, 19711972), Vols. 1-2.

4. G. J. Schultz, Rev. Mod. Phys. 45, 423 (1973).

5. G. F. Drukarev, Collisions of Electrons with Atoms and Molecules (Nauka, Moscow, 1978; Plenum, New York, 1987).

6. Yu. N. Demkov and V. N. Ostrovskii, Zero-Range Potentials and Their Applications in Atomic Physics Plenum, New York, 1988).

7. L. G. Christophorou, Electron Molecule Interaction and Their Application (Academic, New York, 1984).

8. H. S. W. Massey, Negative Ions (Cambridge Univ. Press, Cambridge, 1976; Mir, Moscow, 1979).

9. A. Herzenberg, Electron-Molecular Collision (Plenum, New York, 1984).

10. W. Domcke, Phys. Rep 208, 98 (1991).

11. A. Chutjian, A. Garscadden, and J. M. Wadehra, Phys. Rep. 264, 393 (1996).

12. I. Prigogine and I. Strengers, Quantum, Chaos, Time. To a Solution of the Time Paradox (Editorial URSS, Moscow, 2003).

13. S. P. Merkur'ev and L. D. Faddeev, Quantum Theory of Scattering for Several Particle Systems (Nauka, Moscow, 1985) [in Russian].

14. S. A. Pozdneev, Application of Quantum Theory of Scattering to the Calculating Various Processes in Nuclear, Atomic, and Molecular Physics (Yanus-K, Moscow, 2001) [in Russian].

15. S. A. Pozdneev, Phys. Lett. B 125, 335 (1983); J. Russ. Laser Res. 22, 175 (2001); 38, N2, 107-123, 2017.

16. T. K. Rebane, Yad. Fiz. 61, 61 (1998) [Phys. At. Nucl.61, 56 (1998)].

17. S. A. Pozdneev, Sov. Phys. JETP 50, 18 (1979); JETP 90, 30 (2000);

18. K. P. Huber and G. Herzberg, Molecular Spectra and Molecular Structure, Vol. 4: Constants of Diatomic Molecules (Van Nostrand Reinhold, New York, 1979; Mir, Moscow, 1984).

19. H. Drexel, G. Senn, T. Fiegele, etal., J. Phys. B: At. Mol. Opt. Phys. 34, 1415 (2001).

20. A. P. Hickman, Phys. Rev. 43, 3495 (1991).

21. G. A. Gallup, Y. Xu, and I. I. Fabricant, Phys. Rev. A 57,2596 (1998).

22. V. V. Popyshev, Yad. Fiz. 66, 64 (2003) [Phys. At. Nucl. 66, 61 (2003)]; J. Phys. A: Math. Gen. 36, L13 (2003).

23. A. V. Finkel'shtein and O. B. Ptitsin, Physics of Protein: Course of Lectures (Knizhnyi Dom Universitet, Moscow, 2002) [in Russian].

24. B. N. Zakhar'ev and V. M. Chabanov, Controllable Quantum Mechanics. A New Status of the Theory of the Approach to Inverse Problem (Inst. Komp'yut. Issled., Moscow, 2002) [in Russian].

25. F. M. Pen'kov, JETP 91, 698 (2000); Phys. Rev. 62, 044701-1 (2000);

26. Y. Horacek and W. Domke, Phys. Rev. A 53, 2262 (1996).

27. S. A. Pozdneev, JETP, v.99, N5, 2004, p.915-933.

28. W. Schollkopf and J. P. Toennies, J. Chem. Phys. 104, 1155 (1996); Phys. Rev. Lett. 85, 2284 (2000).

29. D. V. Fedorov and A. S. Jensen, Phys. Rev. C 50, 2372,(1994).

30. A. K. Motovilov, E. A. Kalganova, and S. A. Sofianos, J. Phys. B: At. Mol. Opt. Phys. 31, 1279 (1998); J. Chem. Phys. 275, 168 (1997); Phys. Rev. A 56, R1686 (1997).

31. V. Rudnev and S. Yakovlev, Chem. Phys. Lett. 22, 97 (2000).

32. A. R. Janzen and R. A. Aziz, J. Chem. Phys. 79, 4330,(1979).

33. J.Perez-Rios, S.Ragole, J.Wang and C.H.Greene, J.Chem.Phys., v.140, 044307-1, (2014)

34. V.M.Azriel, L.I.Kolesnicova, L.Yu.Rusin, J.Chem.Phys., v.35, N8, p.3-10, (2016) 


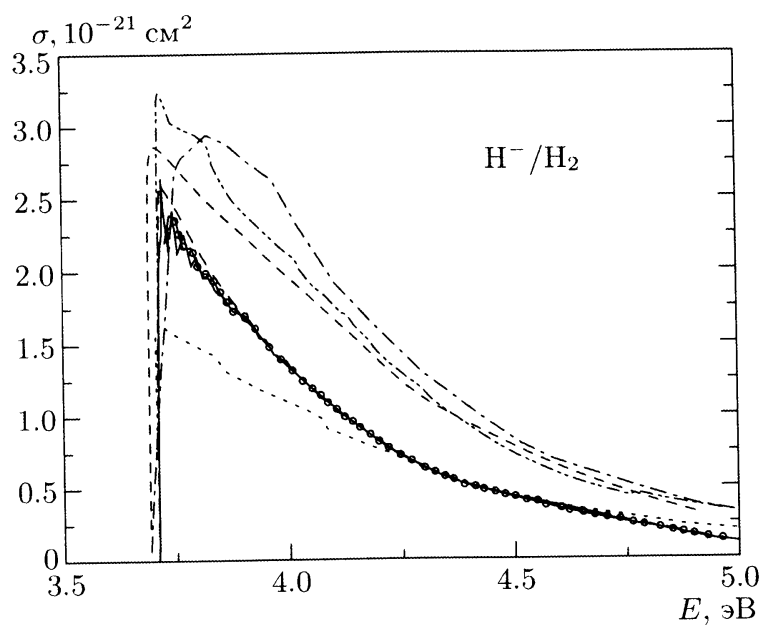

Figure 1. Dependencies of the cross section of electron dissociative attachment to hydrogen molecules on the energy of projectile electrons: the solid curve corresponds to experimental data [19]; the results of calculations performed in [11], [7, 10], [10, 26], [21], and [20] are represented by the fine-dash, large-dash, dot-and-dash, dotted, and double dot-and-dash curves, respectively; our results of calculation are presented as circles. 


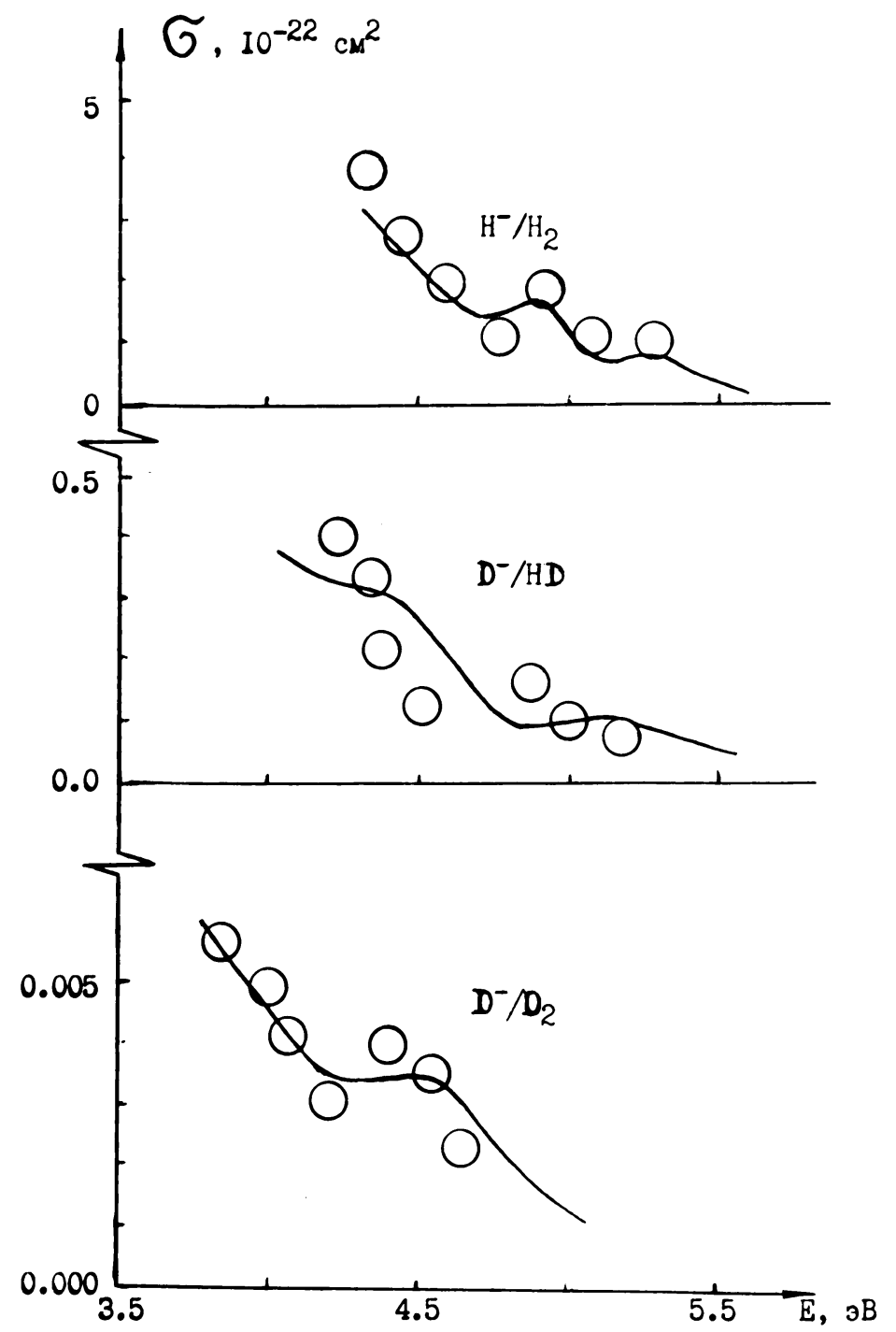

Figure 2. Dissociative attachment of electrons to $H_{2}$ molecules. Region $3.75 \mathrm{eV}$. The solid lines correspond to the results of our calculation, experimental data [4] are presented as circles. 


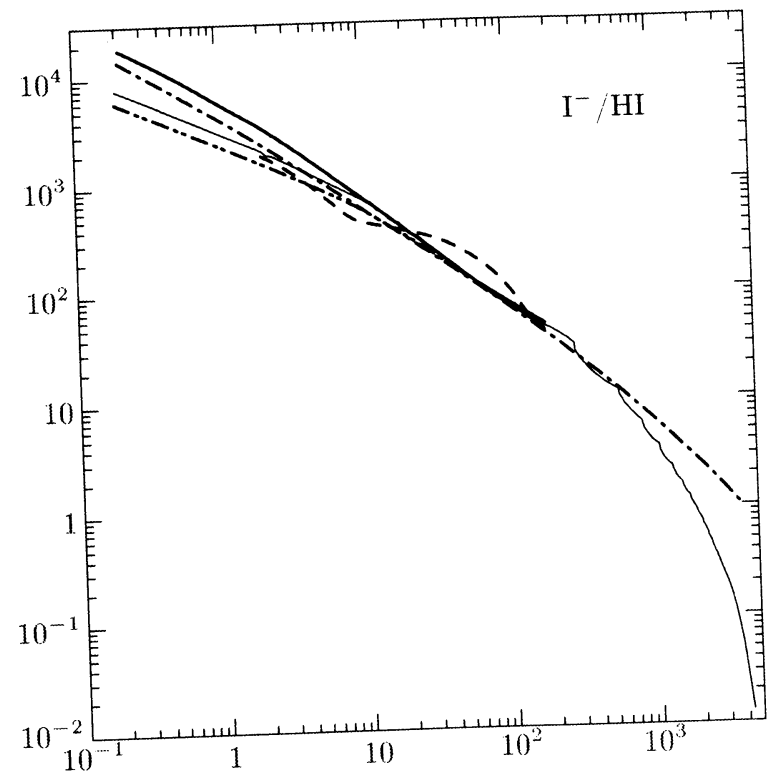

Figure 3. Dissociative attachment of electrons to $H I$ molecules. The dashed, fine solid and bold solid lines correspond to calculations [19], [9] and [21] respectively; our results are shown by dot-and-dash curve. 


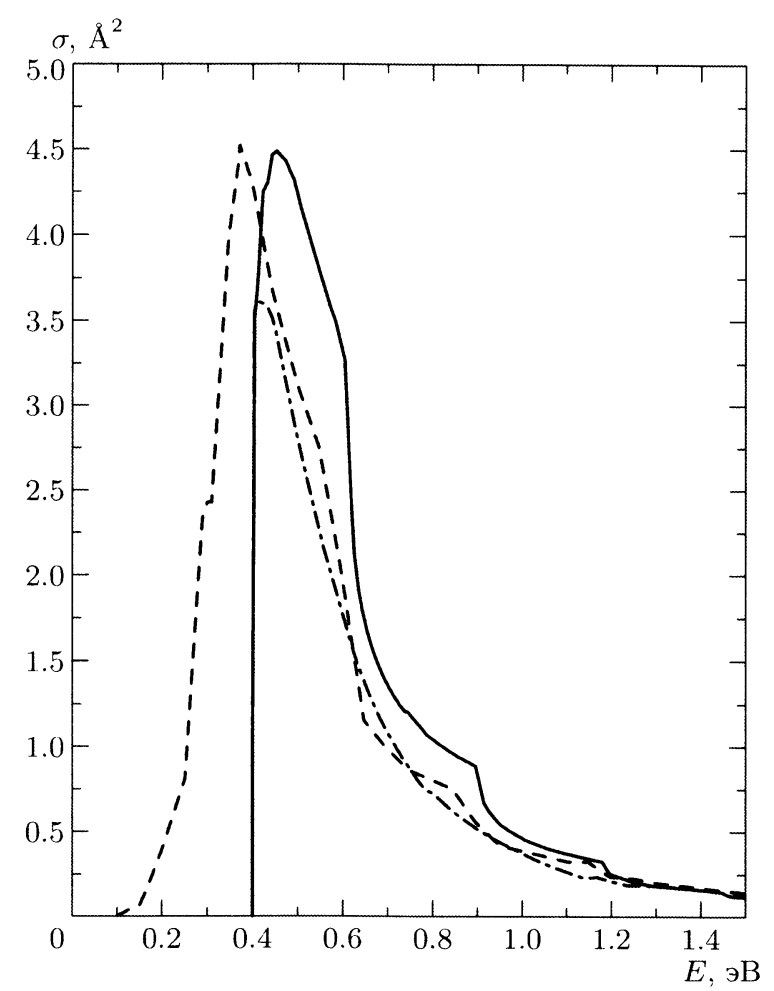

Figure 4. Dissociative attachment of electrons to $\mathrm{HBr}$ molecules. The solid line corresponds to calculations [26]; our results - dot-and-dash curve. 

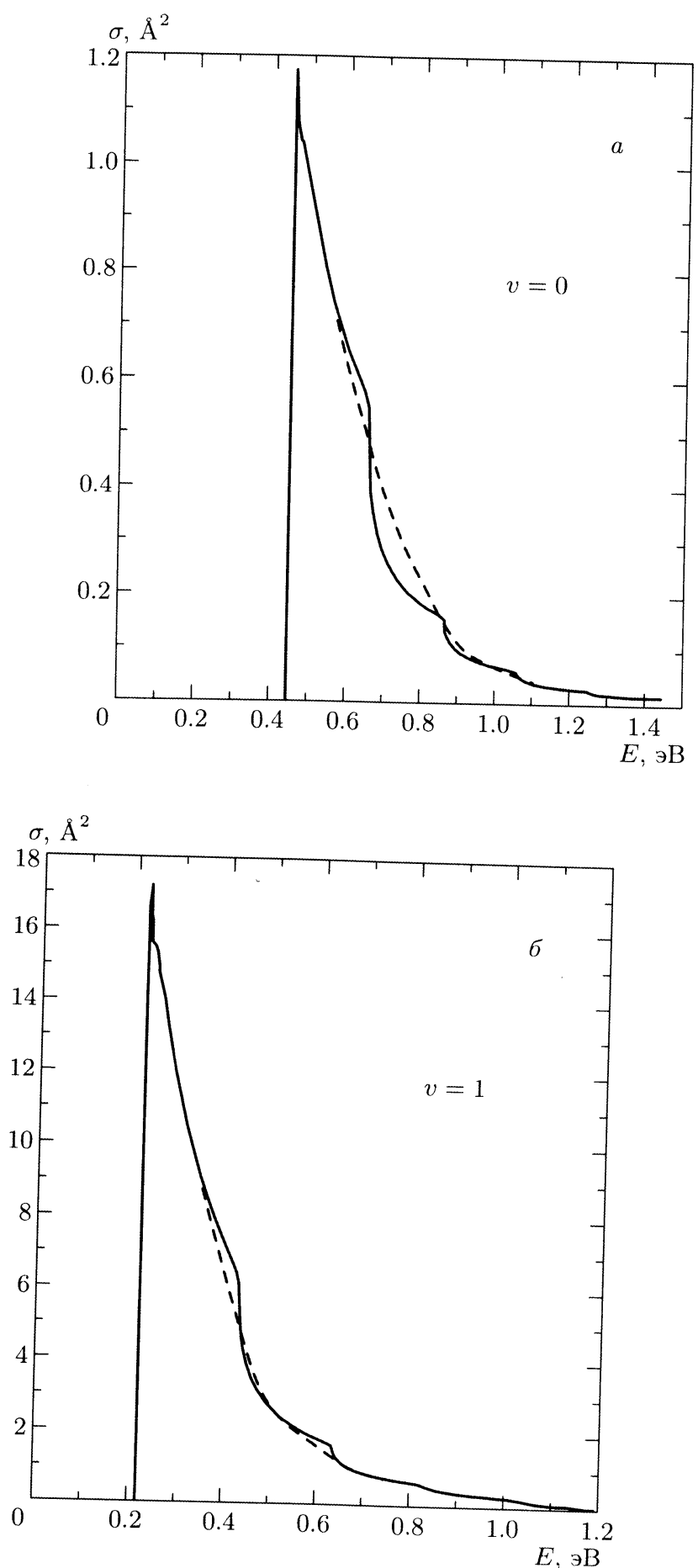

Figure 5. Dissociative attachment of electrons to $\mathrm{HBr}$ molecules that were initially (a) in the ground state $(\mathrm{v}=0)$ and (b) in the exited state $(\mathrm{v}=1)$. The solid line corresponds to the results of calculations performed in [26]; our results - dashed curve. 


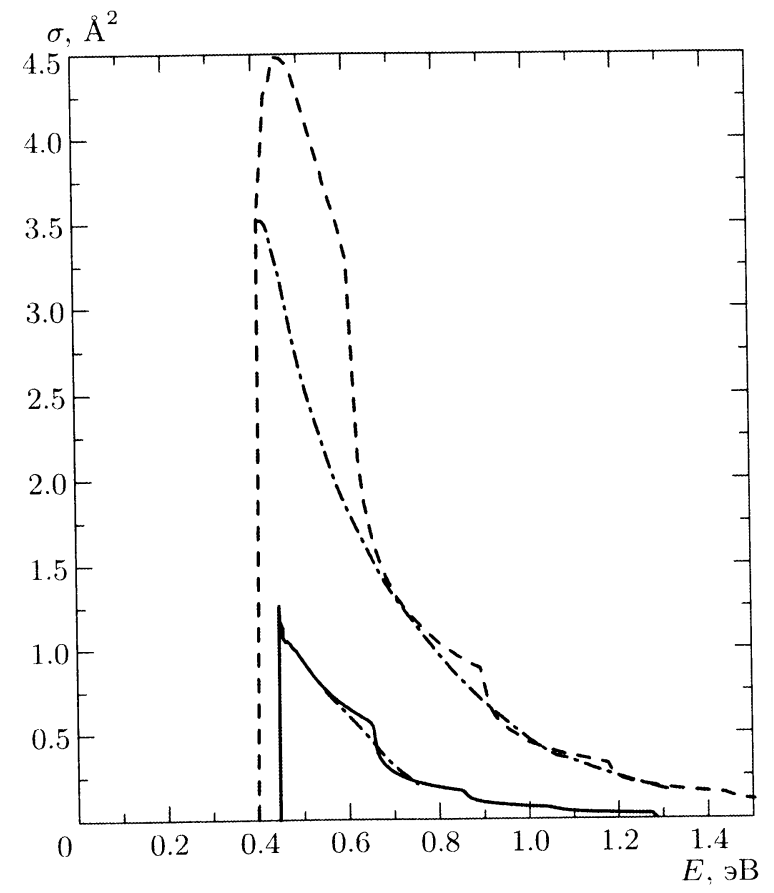

Figure 6. Isotopic effect in dissociative attachment electrons to $H B r$ and $D B r$ molecules. The solid line and dashed curves correspond to the results of calculations performed by using the nonlocal theory [26]; our results dot-and-dash curve.

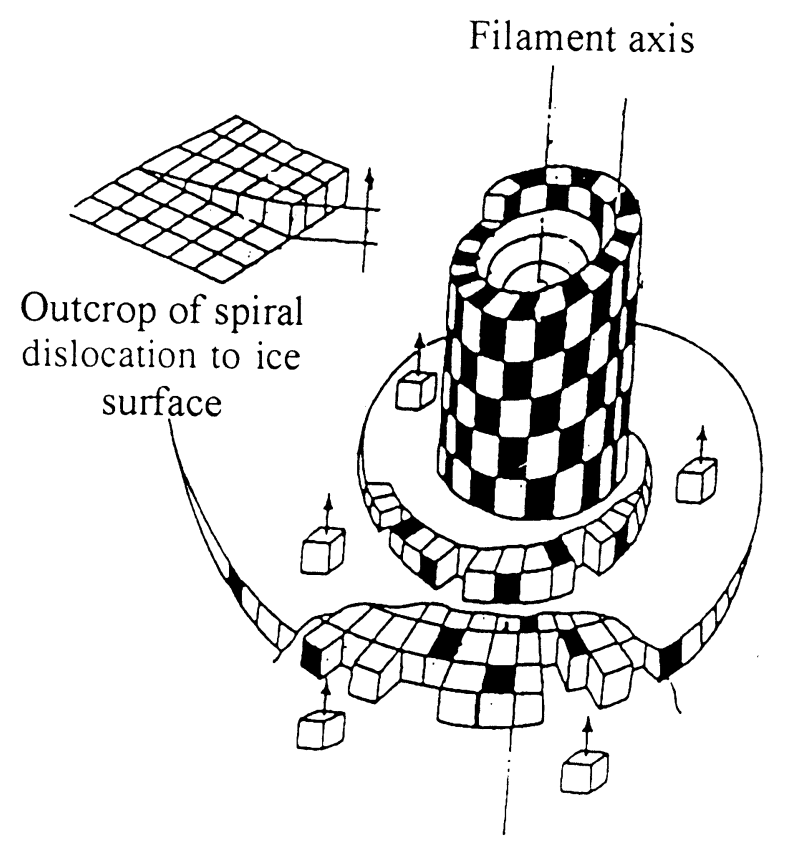

Grown direction

Figure 7. Schematic drawing of spiral type structure growth. 


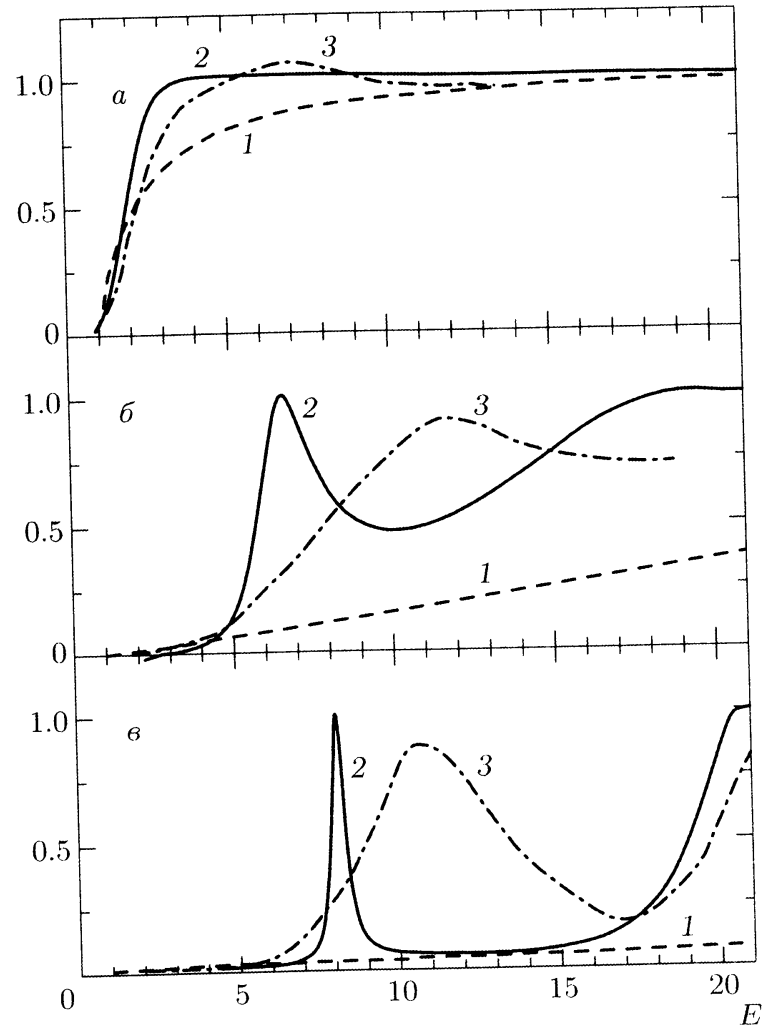

Figure 8. Tunneling probabilities for structureless (1) and structured $(2,3)$ particles as functions of the energy of the projectile particle for the harmonic potential (2) and the Morse potential (3) for various characteristics of the barrier a $=1$ (a), 6 (b), and 11 (c). Energy, E, is given in units of the corresponding potential. 

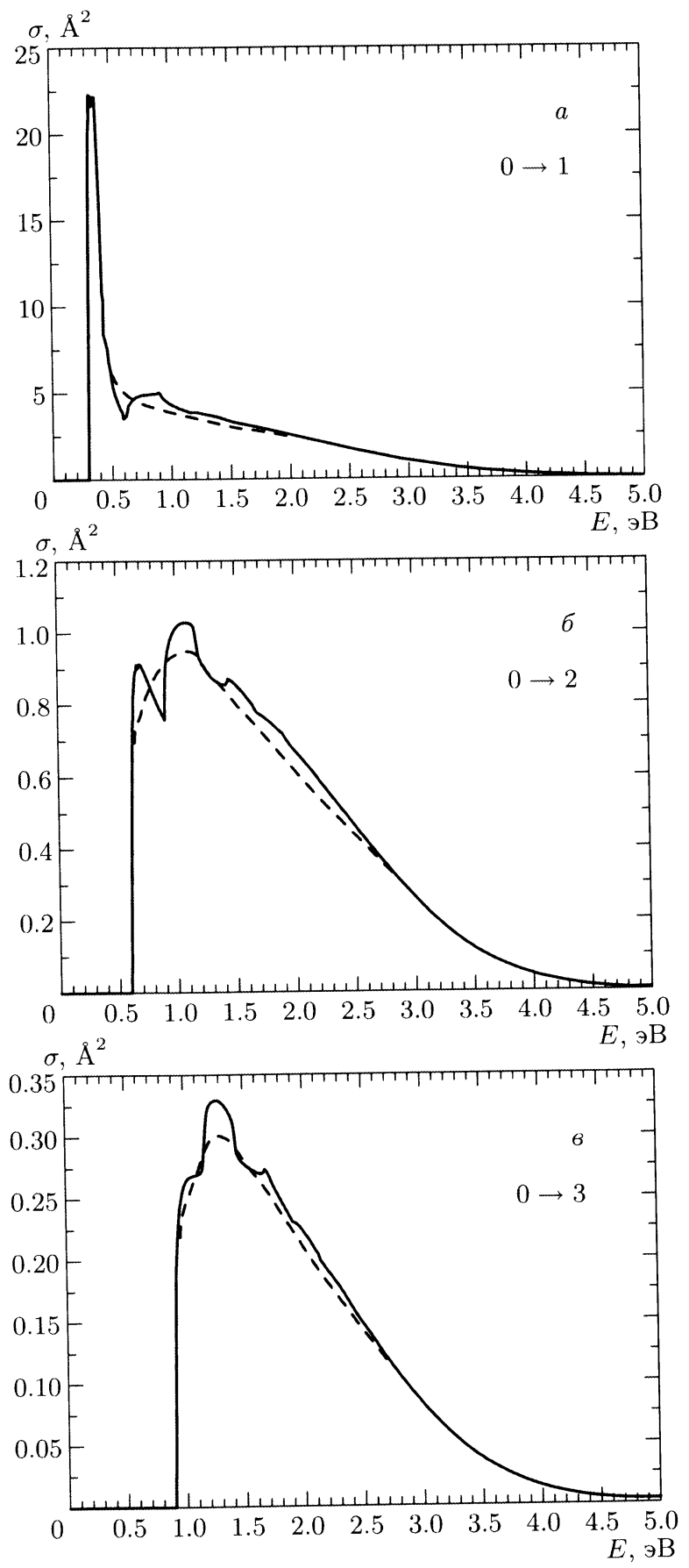

Figure 9. Vibrational excitation of hydrogen halide molecules $(D B r)$ by electrons, $e+D B r(v=0) \rightarrow e+H B r(v=$ 0 ) (a), 2 (b), 3 (c). The dashed curves describe our results and solid curves show the results of calculations performed in $[26]$. 

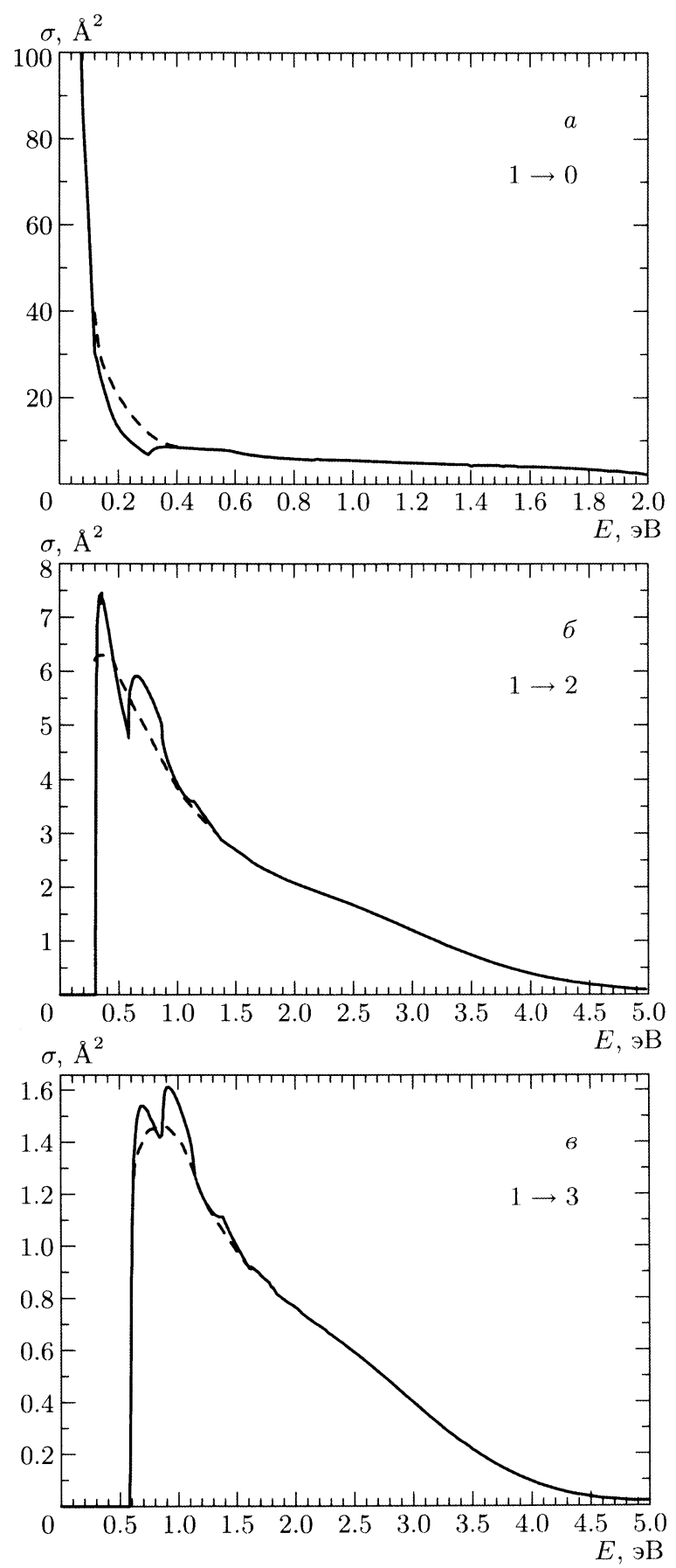

Figure 10. Vibrational excitation of $H B r$ molecules by electrons, $e+H B r(v=1)$ (a), 2 (b), 3 (c). The dashed curve describes our results and solid curves show the results of calculations performed in [26]. 

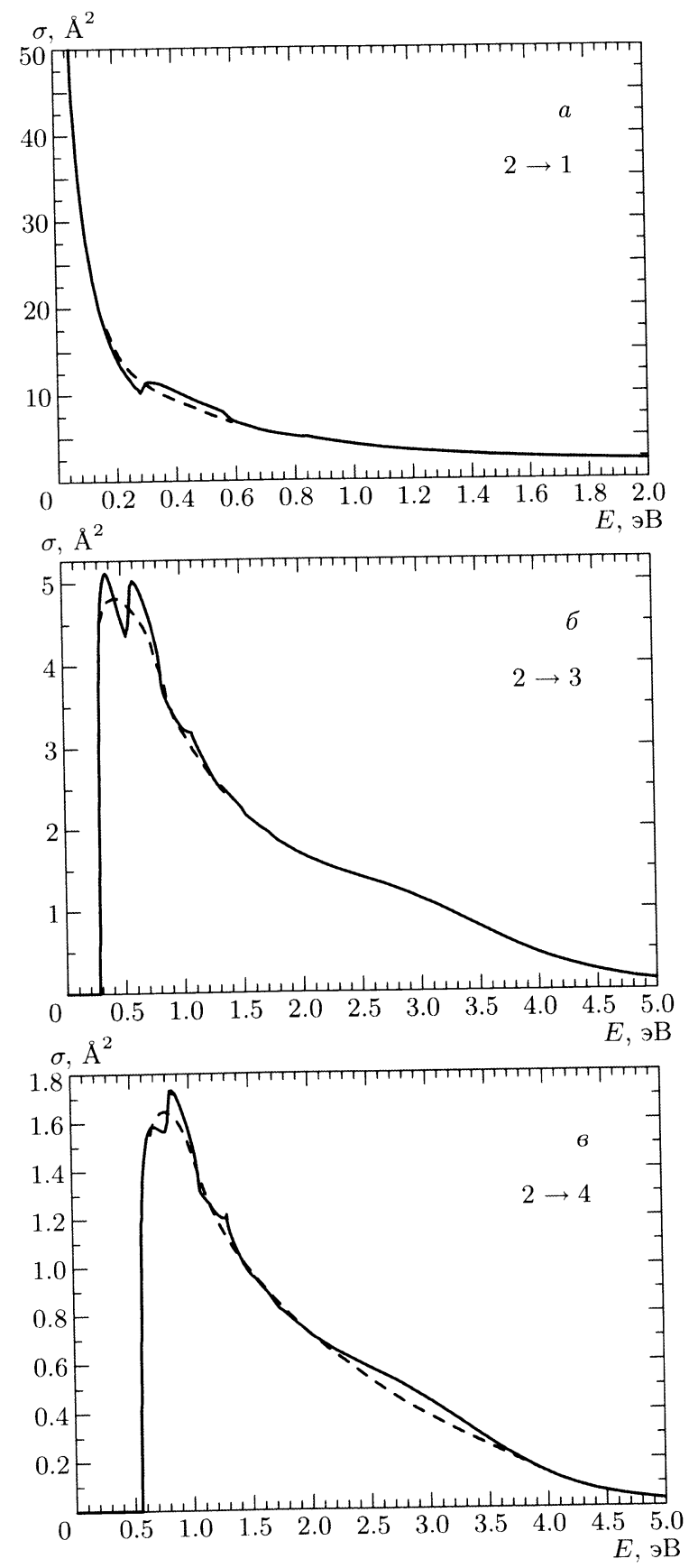

Figure 11. Vibrational excitation of $H B r$ molecules by electrons $e+H B r(v=2)$ (a), 2 (b), 3 (c). . The dashed curve describes our results and solid curves show the results of calculations performed in [26]. 

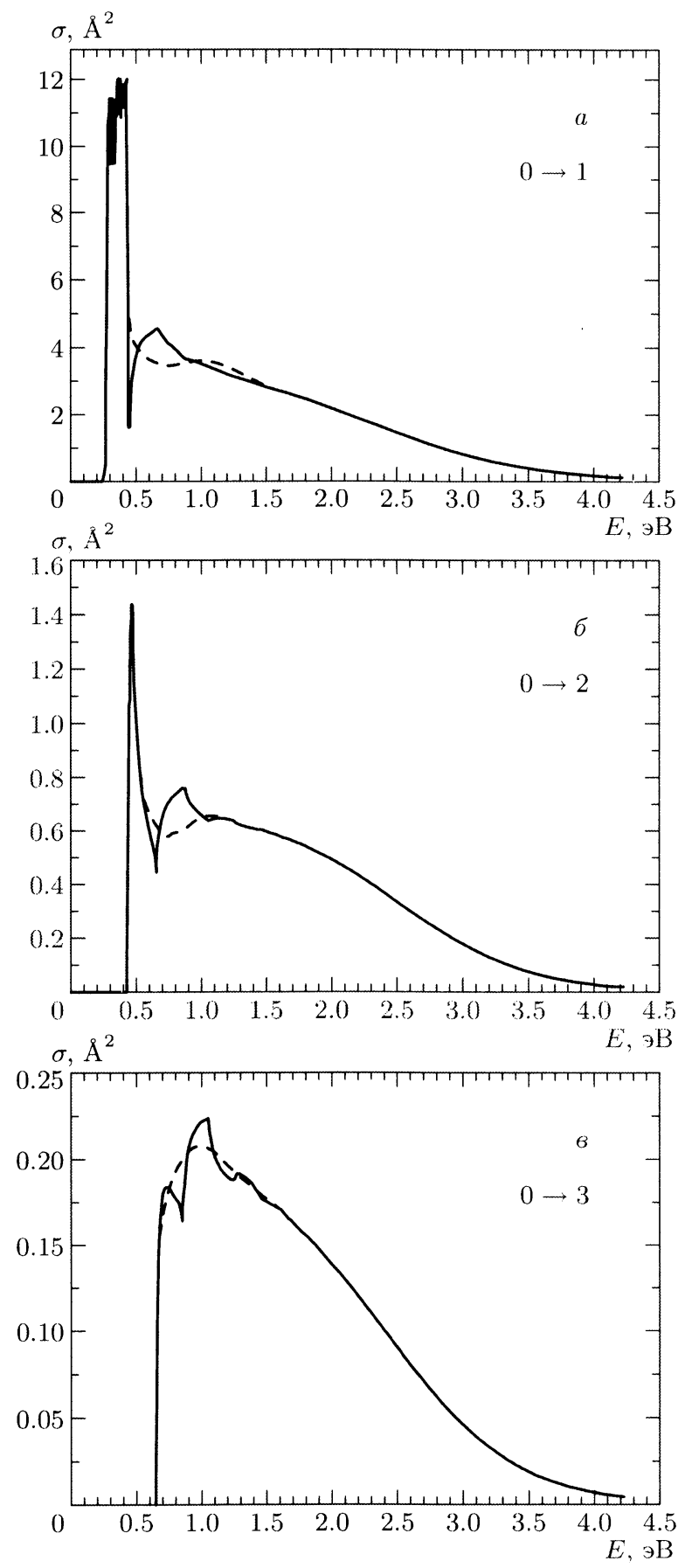

Figure 12. Vibrational excitation of $D B r$ molecules by electrons $e+D B r(v=0)$ (a), 2 (b), 3 (c). . The dashed curve describes our results and solid curves show the results of calculations performed in [26]. 


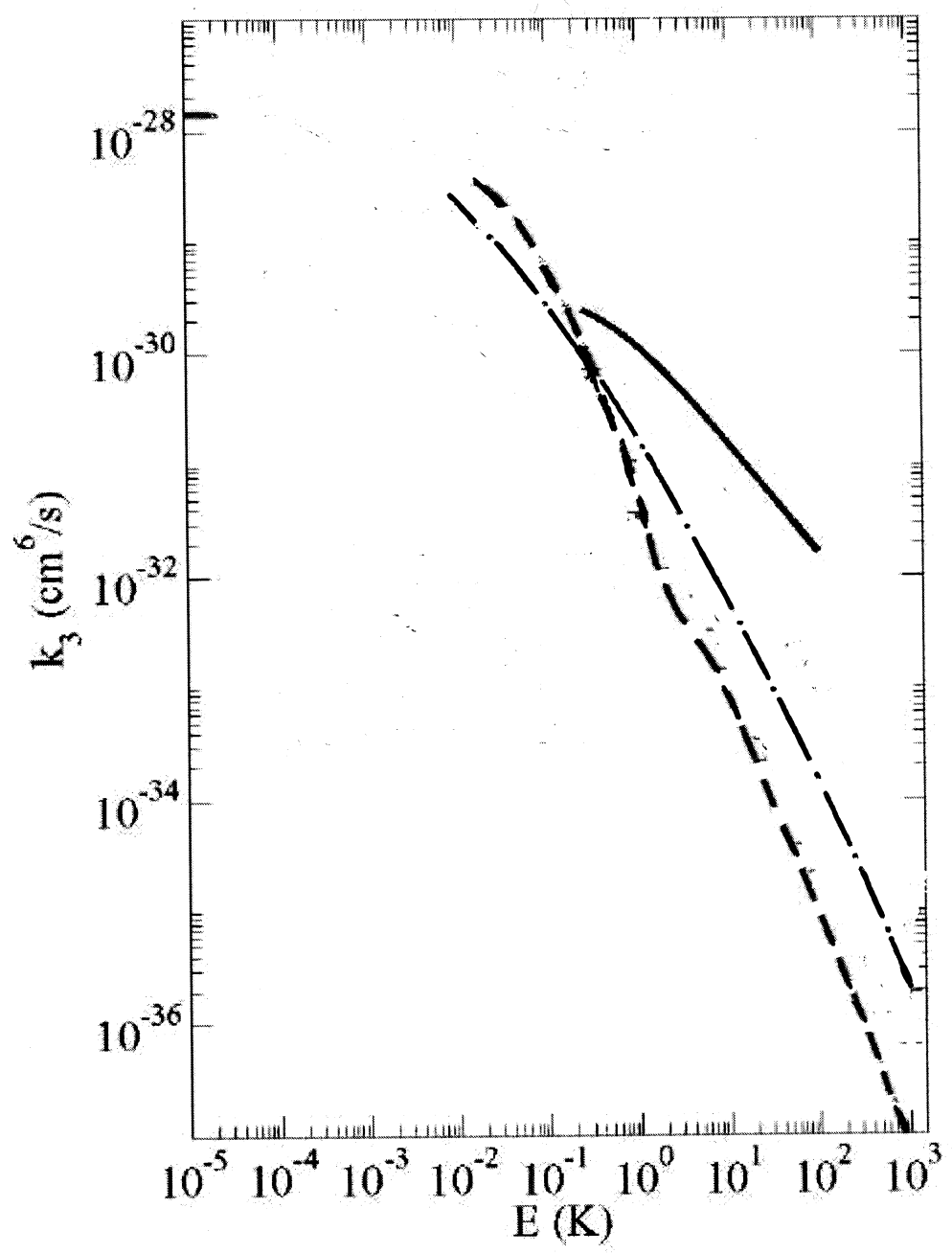

Figure 13. The three-body recombination rate of helium atoms. - - - - - present calculation; - - - classical trajectory calculations [33]; -. - . - .- - classical trajectory calculations for fixed angular momentum [29-31]. 


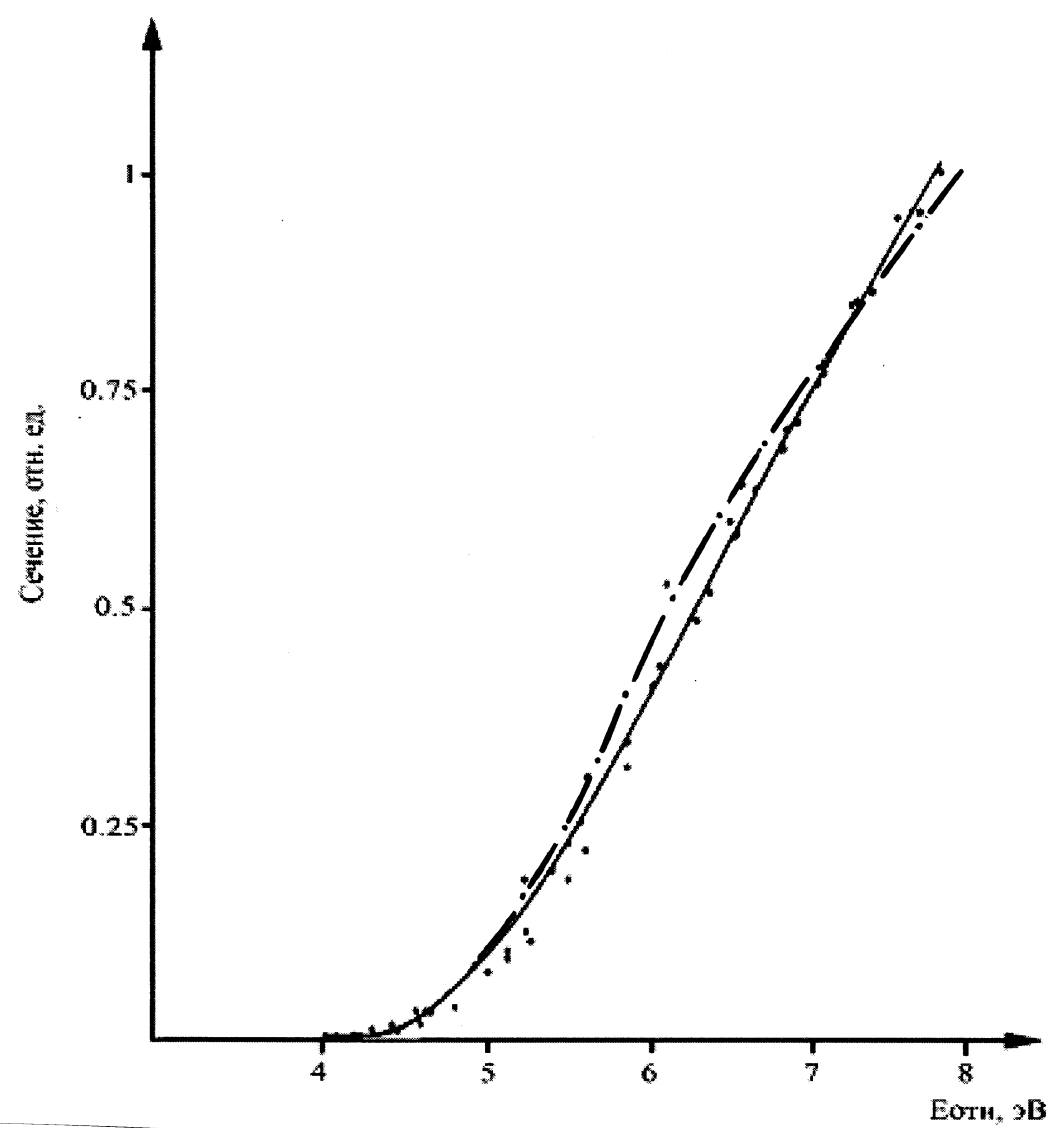

Figure 14. Calculations of the reaction $\mathrm{CsBr}+\mathrm{Xe} \rightarrow \mathrm{Cs}^{+}+\mathrm{Br}^{-}+\mathrm{Xe} .-----$ present calculation; -. - . - .- - classical trajectory calculations [34]; ..... - experimental data [32-34]. 


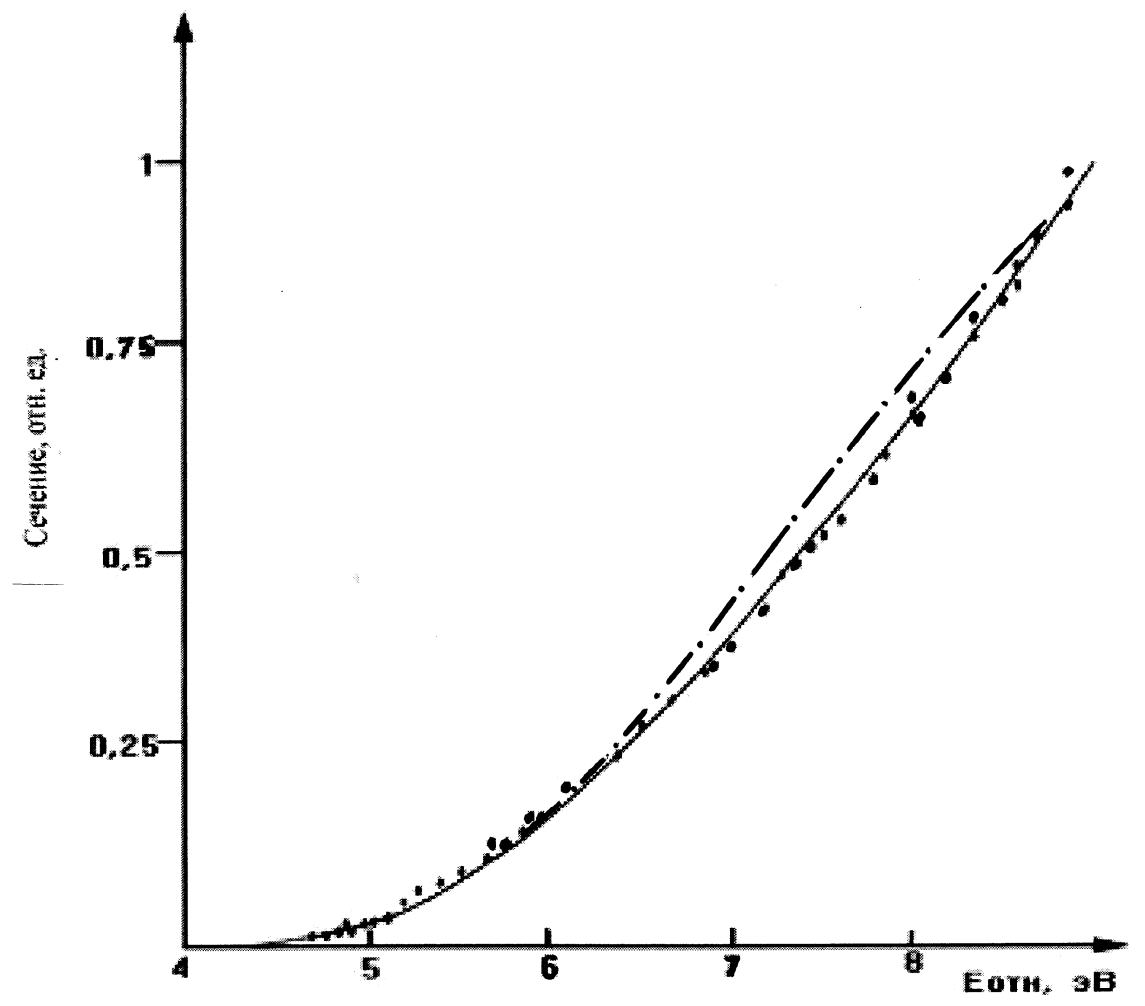

Figure 15. Calculations of the reaction $\mathrm{CsBr}+\mathrm{Hg} \rightarrow \mathrm{Cs}^{+}+\mathrm{Br}^{-}+\mathrm{Hg} .-----$ present calculation; -. - . - .- - classical trajectory calculations [33]; ..... - experimental data [32-34]. 